\title{
New perspectives on the renal slit diaphragm protein podocin
}

\author{
Manfred Relle ${ }^{1,4}$, Hannes Cash ${ }^{2,4}$, Christoph Brochhausen ${ }^{3}$, Dennis Strand ${ }^{1}$, Julia Menke ${ }^{1}$, \\ Peter R Galle ${ }^{1}$ and Andreas Schwarting ${ }^{1}$ \\ ${ }^{1}$ Department of Medicine, Johannes Gutenberg University, Mainz, Germany; ${ }^{2}$ Department of Urology, \\ Charité-University Medicine, Berlin, Germany and ${ }^{3}$ Institute of Pathology, Johannes Gutenberg University, \\ Mainz, Germany
}

Podocin is a critical component of the glomerular filtration barrier, its mutations causing recessive steroidresistant nephrotic syndrome. A GenBank analysis of the human podocin (NPHS2) gene resulted in the possible existence of a new splice variant of podocin in the kidney, missing the in-frame of exon 5 , encoding the prohibitin homology domain. Using RT-polymerase chain reaction and immunoblotting followed by sequence analysis, we are for the first time able to prove the expression of a novel podocin isoform (isoform 2), exclusively and constitutively expressed in human podocytes. Furthermore, we reveal singular extrarenal podocin expression in human and murine testis. Our data show the Sertoli cells of the seminiferous tubules to be the origin of testicular podocin. Confocal laser microscopy illustrates the co-localization of podocin with filamentous actin within Sertoli cells, suggesting a role of podocin in the blood/testis barrier. These results led to the rationale to examine podocin expression in testes of men with Sertoli cell-only syndrome, a disorder characterized by azoospermia. Interestingly, we observed a complete down-regulation of podocin mRNA in Sertoli cell-only syndrome, indicating a possible role of podocin in the pathogenesis of this germinal aplasia. Men with Sertoli cell-only syndrome show normal renal podocin expression, suggesting an alternate regulation of the testicular promoter. Our findings may change the perception of podocin and give new insights into the ultrastructure of glomerular slit diaphragm and the blood/testis barrier.

Modern Pathology (2011) 24, 1101-1110; doi:10.1038/modpathol.2011.58; published online 15 April 2011

Keywords: isoform; kidney; podocin; Sertoli cell-only syndrome; Sertoli cells; testis; WT-1

Podocytes are cells of the glomerular visceral epithelium in the kidney, covering the basement membrane of glomerular capillaries as a continuous layer. These specialized, terminally differentiated epithelial cells consist of a cell body and foot processes, which interdigitate with the foot processes of neighboring podocytes, creating what is known as the slit diaphragm. Terminal differentiation of podocytes is regulated by WT-1, a transcription factor that is expressed in podocytes and thus serves as a specific marker for these cells. ${ }^{1-3}$ The stability of the slit diaphragm is sustained by multiple molecular interactions that ensure stable

Correspondence: Dr M Relle, MS, First Department of Medicine, University Hospital Johannes-Gutenberg University Mainz, Langenbeckstrasse 1, Mainz D-55131, Germany.

E-mail: relle@uni-mainz.de

${ }^{4}$ These two authors are the co-first authors.

Received 18 January 2011; revised 23 February 2011; accepted 23

February 2011; published online 15 April 2011 anchorage of the membrane complexes to the actin cytoskeleton. ${ }^{4}$ One of these interacting structural proteins is called podocin (NPHS2). Podocin, a 42$\mathrm{kDa}$ membrane protein, is located on the podocyte foot process where it's $\mathrm{COOH}$ terminus interacts with the transmembrane protein nephrin ${ }^{5}$ and CD2associated protein. ${ }^{6}$ Podocin accumulates in an oligomeric form in lipid rafts, specialized membrane microdomains, where sphingolipids and cholesterol are highly enriched. ${ }^{6,7}$ Boute et $a l^{8}$ were the first to show that mutations of the NPHS2 gene, which encodes podocin, are responsible for a subset of childhood steroid-resistant nephrotic syndrome with focal segmental glomerulosclerosis. In all, $20-40 \%$ of the familial, autosomal recessive, pediatric steroid-resistant nephrotic syndrome can be ascribed to mutations of the NPHS2 gene. ${ }^{9}$

Although podocin is believed to be exclusively expressed in the glomerular podocytes, ${ }^{10}$ an extrarenal expression of podocin has been implied. ${ }^{8}$ Until now, no proof has been provided. When 
looking at other structural proteins of the slit diaphragm, nephrin, also believed to be solely expressed on glomerular podocytes, is found in extrarenal murine tissues such as testis and brain. ${ }^{11}$ Densin, another structural protein of the slit diaphragm, was proven to be expressed in human and murine podocytes, brain and testis. ${ }^{12}$ In the testis, both proteins seem to be localized and expressed in the cell membrane of Sertoli cells. ${ }^{11}$ Besides the possible extrarenal manifestation of podocin, the existence of a podocin isoform has been discussed, but direct evidence has not yet been provided..$^{13}$ These two assumptions were the basis for our new study. Our intention was to clarify the existence of a podocin isoform and the extrarenal podocin expression in human tissue.

\section{Materials and methods}

\section{Tissue Sample Preparation}

Immediately following surgical removal, the tissues were embedded in Tissue-Tek OCT medium (Sakura, Tokyo, Japan) and stored at $-80^{\circ} \mathrm{C}$ until used in immunohistology or directly processed for immunoblotting.

Tissue lysates for western blot were purchased from Clontech Laboratories (Palo Alto, CA, USA; testis, spermatic cord, cerebrum, liver and lung) or isolated from unaffected parts of human renal tumor nephrectomy specimen (whole kidney and glomerular epithelial cells, see below).

All human RNA samples for RT-polymerase chain reaction (PCR) analysis were purchased from Biochain Institutes (Hayward, CA, USA; cerebrum (24-year-old male), adult kidneys (26- and 46-yearold males, and 68-year-old female), pancreas (62year-old male) and testis (29-year-old male) normal total RNAs) or from Invitrogen (Karlsruhe, Germany; total RNA from poorly differentiated renal clear cell carcinoma (35-year-old female), adult (26-year-old healthy male) and fetal kidney mRNA (pool of 28- and 32-week-old male and female)).

\section{RNA Isolation from Mouse Tissues}

Kidneys, testes and livers from FVB mice were snap frozen in liquid nitrogen. Total RNA was isolated using the reagents and protocols included in the RNeasy Lipid Tissue kit (Qiagen, Hilden, Germany). RNA quantity and quality were determined spectrophotometrically at 260 and $280 \mathrm{~nm}$.

\section{Primer Design and Touch-Down RT-PCR}

Based on GenBank accessions, PCR primers $\left(5^{\prime}-3^{\prime}\right.$ direction) were designed as follows: human podocin (sense: GCC-CTG-CCT-GGA-TAC-CTA-CCA-CAA, antisense: TTC-AGC-CTC-CAC-AGC-CAG-TGA-GTG), human glyceraldehyde-3-phosphate dehydrogenase
(GAPDH) (sense: GTC-TTC-ACC-ACC-ATG-GAG-A AG-GCT, anti-sense: CAT-GCC-AGT-GAG-CTT-CCCGTT-CA), human $\beta$-2 microglobulin (sense: AGCGTA-CTC-CAA-AGA-TTC-AGG-TT, anti-sense: TA C-ATG-TCT-CGA-TCC-CAC-TTA-ACT-AT), murine podocin (sense: ACC-CTG-TCT-GGA-CAC-CTA-TC A-CAA-G, anti-sense: CTC-AGC-TTC-CAC-CGC-C AG-AGA-GTG). Either $200 \mathrm{ng}$ mRNA or $1 \mu \mathrm{g}$ of total RNA from human and murine tissues were converted to cDNA with the SuperScript ${ }^{\mathrm{TM}}$ First-Strand Synthesis System kit (Gibco BRL, Gaithersburg, MD, USA), following the manufacturer's recommendations. The touch-down PCR was performed using $200 \mathrm{ng}$ of cDNA with the following temperature profile: $90 \mathrm{~s}$ at $94^{\circ} \mathrm{C}, 10$ cycles of $30 \mathrm{~s}$ at $94^{\circ} \mathrm{C}, 30 \mathrm{~s}$ at $71^{\circ} \mathrm{C}\left(-1^{\circ} \mathrm{C}\right.$ per cycle), $90 \mathrm{~s}$ at $72^{\circ} \mathrm{C}$, followed by 25 cycles of $30 \mathrm{~s}$ at $94^{\circ} \mathrm{C}, 30 \mathrm{~s}$ at $60^{\circ} \mathrm{C}$ and $90 \mathrm{~s}$ at $72^{\circ} \mathrm{C}$. The resulting DNA fragments were separated by $1.3 \%$ agarose gel electrophoresis stained with ethidium bromide.

\section{Sequence Analysis and Identification of PCR Products}

The separated PCR products from kidney and testis were purified using a QIA quick gel extraction kit (Qiagen). Purified PCR products were subjected to direct sequencing by GENterprise (Mainz, Germany) using an automated DNA sequencer ABI 373A (Applied Biosystems). Sequences were subjected to BLAST analysis and were checked for homology with podocin DNA sequences.

Computational splice site prediction of the exons 4, 5 and 6 from the human NPHS2 gene (GenBank accession number: NG_007535.1) was performed using a combination of the online tools NetGene2 (http://www.cbs.dtu.dk/services/NetGene2) ${ }^{14}$ and ASSP (Alternative splice site predictor; http:// www.es.embnet.org/ mwang/assp.html). ${ }^{15}$

\section{Specificity of the Podocin Primary Antibodies}

For the immunohistological detection of human podocin, a rabbit anti-Podocin IgG peptide antibody was used, which reacts with amino-acid residues 367-383 of human podocin (Sigma-Aldrich, St Louis, MO, USA). Rabbit anti-human podocin antibody P35 was a gift from Dr C Antignac. ${ }^{16}$ It is raised against the C-terminal region downstream of the membraneassociated domain (amino acids 135-383).

\section{Immunoblotting of Glomerular and Whole Kidney Lysates}

Whole kidney cells and glomerular epithelial cells were isolated from unaffected parts of human renal tumor nephrectomy specimen with the use of $0.15 \%$ collagenase type IV (Sigma, St Louis, MO, USA) according to our protocol ${ }^{17}$ and solubilized on ice with RIPA buffer plus protease inhibitors $(1 \times \mathrm{PBS}$, $1 \%$ Nonidet P-40, $0.5 \%$ sodium deoxycholate, $0.1 \%$ 
SDS, $10 \mu \mathrm{g} / \mathrm{ml}$ PMSF, $30 \mu \mathrm{g} / \mathrm{ml}$ aprotinin). The detergent extract was centrifuged (16000 g, $20 \mathrm{~min}$ ) and the supernatant was collected.

Frozen renal tissue was diced into small pieces and thawed in ice-cold RIPA buffer. Homogenization was performed with the Ultra-Turrax rotor-stator device at $4^{\circ} \mathrm{C}$. Lysates were incubated for $30 \mathrm{~min}$ on ice, centrifugated (10000 g, $20 \mathrm{~min}$ ) and the total cell lysates were collected.

Glomerular and kidney lysates were quantified with a Bradford protein assay (Bio-Rad Laboratories, Hercules, CA, USA) and $25 \mu \mathrm{g}$ of each sample was subjected to SDS-polyacrylamide gel electrophoresis and immunoblotting as described. ${ }^{18}$ The membrane was incubated with anti-human podocin antibody p35 $(1 \mathrm{ng} / \mu \mathrm{l})$ for $2 \mathrm{~h}$ and with horseradish peroxidase-conjugated goat anti-rabbit IgG (diluted 1:2500) for $1 \mathrm{~h}$ at RT. Bound antibodies were visualized by the Lumi-Light Plus Western Blotting Substrate (Roche, Mannheim, Germany) and a subsequent exposure to a Kodak BioMax Light Film (Eastman Kodak, Rochester, NY, USA).

\section{Immunostaining}

Human renal and testicular tissue was obtained from unaffected parts of human renal tumor nephrectomy specimen and from negative testicular biopsies. Podocin expression in human kidneys and testes was analyzed by immunohistochemistry of acetone-fixed, frozen sections and paraffin sections. For frozen sections, rabbit anti-podocin IgG peptide antibody ( $5 \mathrm{ng} / \mu \mathrm{l}$; Sigma-Aldrich) or normal rabbit IgG (R\&D Systems, Wiesbaden, Germany) was added and incubated for $2 \mathrm{~h}$. The secondary antibody used was biotinylated goat anti-rabbit IgG (1:4000; Vector Laboratories, Burlingame, CA, USA). After $1 \mathrm{~h}$ at $4^{\circ} \mathrm{C}$, detection was performed using the Vectastain Elite ABC kit (Vector Laboratories) and DAB color development (DAKO, Hamburg, Germany). For paraffin sections, the primary antibodies were used in a dilution of 1:50 (Podocin, Sigma-Aldrich) and 1:200 (WT-1, Santa Gruz, USA). The immunohistochemistry was performed automatically according to standard methods with help of the DAKO autostainer (DAKO) by use of proteinase $\mathrm{K}$ pretreatment and DAB or APPAP, respectively, as chromogen. After staining, the sections were mounted and staining was evaluated by light microscopy (Olympus BX45, Hamburg, Germany).

\section{Confocal Laser Scanning Microscopy}

Paraformaldehyde-fixed frozen sections of human kidney and testis were permeabilized with $0.1 \%$ Triton X-100 in PBS. After blocking (20\% goat serum and $10 \% \mathrm{BSA}$ in PBS) for $30 \mathrm{~min}$, the slides were incubated with rabbit anti-Podocin $\operatorname{IgG}(5 \mathrm{ng} / \mu \mathrm{l}$; Sigma-Aldrich) or control rabbit IgG antibody $(5 \mathrm{ng} / \mu \mathrm{l})$ at $4^{\circ} \mathrm{C}$ for $1 \mathrm{~h}$. Subsequently, the sections were reacted to an Alexa Fluor 488-conjugated goat anti-rabbit IgG (1:200; Molecular Probes, Eugene, OR, USA) at RT for $1 \mathrm{~h}$. Filamentous actin was then labeled with Texas Red X-conjugated phalloidin (diluted 1:100). Sections were mounted on glass slides with DAPI-containing anti-fade (Vectashield, Vector Laboratories) and evaluated with a Zeiss 510 confocal laser scanning microscope equipped with an UV-laser (Carl Zeiss, Goettingen, Germany).

\section{Quantitative Gene Expression Analysis}

Total testis RNA was extracted with RNeasy columns (Qiagen) from healthy men and from five azoospermic men, diagnosed with pure Sertoli cellonly syndrome. Podocin-, WT-1- and $\beta 2$-microglobulin-mRNA levels of these probes were quantified by real-time PCR using the QuantiTect SYBR Green RT-PCR kit in combination with sequence-specific QuantiTect Primer Assays (both Qiagen). Gene expression levels were analyzed on an iCycler iQ5 Real-Time PCR Detection System (Bio-Rad Laboratories) under the following conditions: $30 \mathrm{~min}, 50^{\circ} \mathrm{C}$ (reverse transcription), $15 \mathrm{~min}, 95^{\circ} \mathrm{C}$ and 45 cycles of $94^{\circ} \mathrm{C}, 15 \mathrm{~s}$ (denaturation), $56^{\circ} \mathrm{C}, 30 \mathrm{~s}$ (annealing) and $72^{\circ} \mathrm{C}$ for $30 \mathrm{~s}$ (extension). Fluorescence data were analyzed with the iQ5-specific analysis software (version 3.1) and normalized using the housekeeping gene $\beta 2$ microglobulin.

\section{Statistical Analyses}

The Mann-Whitney's U-test was carried out using the WinSTAT ${ }^{\circledR}$ program (Version 2007.1; ${ }^{\circ}$ Robert K. Fitch) for Microsoft Excel $2007^{\circledR}$. P-values below 0.05 were considered statistically significant.

\section{Results}

Podocin mRNA Expression and Sequence Analysis Reveals the Existence of a Podocin Isoform and Proves Singular Extrarenal Expression of Podocin in Human Testis

Gene Bank analysis revealed two different types of podocin (NPHS2) cDNA sequences: one full-length sequence (NM_014625) and one shorter splice variant lacking exon 5 (BC029141). The bioinformatical analysis of the shorter podocin clone showed it to be a result of the skipping of exon 5 ( $\Delta$ exon5), as previously indicated by Horinouchi et $a l,{ }^{13}$ but which has not yet been proven. In order to study the existence of a podocin isoform, we created podocin-specific primers, which were able to amplify both isoforms in one PCR run. Semiquantitative RT-PCR analysis showed strong podocin expression in fetal and adult human kidney as well as in human testis (Figure 1a and c). There was no podocin detection in the pancreas, brain or renal clear cell carcinoma. Since renal podocin is exclusively expressed by podocytes, the negative result in 
clear cell carcinoma was predictable due to its origin from proximal tubular epithelial cells. In addition to the expected podocin band at $382 \mathrm{bp}$, a defined second band at approximately $180 \mathrm{bp}$ was detected in fetal and adult kidney. Three healthy kidney specimens from different healthy individuals obtained from unaffected parts of human renal tumor nephrectomy were used to clarify the question if the second band represents an allelic variant or an alternative isoform. All of the three kidneys showed the same constitutive expression pattern (Figure 1b). To confirm the data revealed by RT-PCR, the different PCR products were purified, sequenced and analyzed. Sequence analysis confirmed that the longer PCR product, found in kidney and testis, was indeed podocin. The shorter product, only found in fetal and adult kidney, had a length of $178 \mathrm{bp}$ and proved to be a podocin isoform lacking exon 5, encoding for the central part of the prohibitin homology domain. The skipping of this in-frame exon (coding for $204 \mathrm{bp}$ ) resulted in a shorter podocin variant, missing 68 amino acids, which we named podocin isoform 2 (Figure 2).

Computational splice site prediction using the online tools NetGene2 and ASSP did not reveal an apparent reason for the skipping of exon 5. Both programs predicted the $5^{\prime}$ and $3^{\prime}$ splice sites of exon 5, demonstrating that the exon borders are not unusual. In addition, the donor and acceptor splice sites of the introns 4 and 5 (compassing exon 5) are in accordance with the GT-AG consensus rule for splicing. ${ }^{19}$

In order to clarify whether the isoform 2 is specific for humans, an RT-PCR analysis of murine (mouse and rat) kidney and testis was accomplished. Both tissues also showed a strong podocin expression, but isoform 2 could not be detected in murine kidney (Figure 1d; Supplementary Figure 1).

\section{Immunoblotting Proves Podocin Expression in Human Kidney and Testis}

The $\alpha$-podocin anti-serum P35 was used to detect podocin in tissue lysates of brain, spermatic cord, testis, glomerulus, whole kidney, liver and lung. We were able to verify our RT-PCR results and demonstrate podocin isoform 1 expression in the lysates of kidney and testis, which can be seen at a band of $42 \mathrm{kDa}$. The second immunoblotting signal at approximately $35 \mathrm{kDa}$ (representing podocin isoform 2) was exclusively found in the glomerular lysate. The other extrarenal tissues showed no evidence of either isoform (Figure 3). As demonstrated at the mRNA level, the immunoblot data also indicated that podocin isoform 1 showed a stronger glomerular signal than isoform 2. Using a molecular mass calculator (http://www.innovagen.se/custompeptide-property-calculator/peptide-property-calculator/peptide-property-calculator.asp), we evaluated the theoretical molecular mass for both podocin isoforms. The theoretical mass of isoform 1 and 2

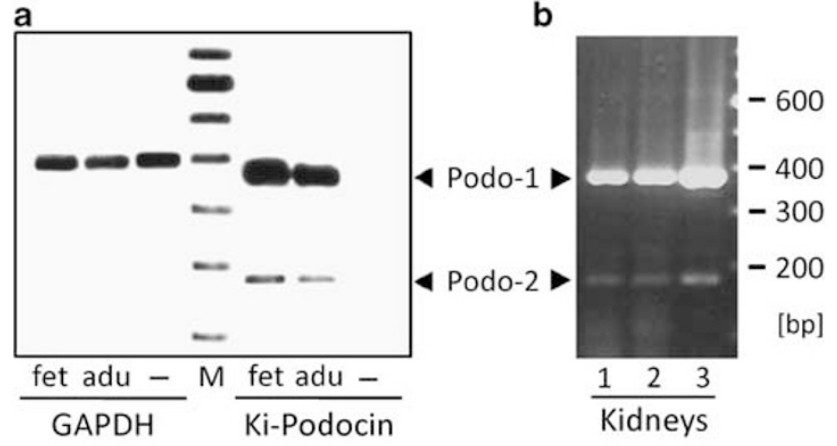

C

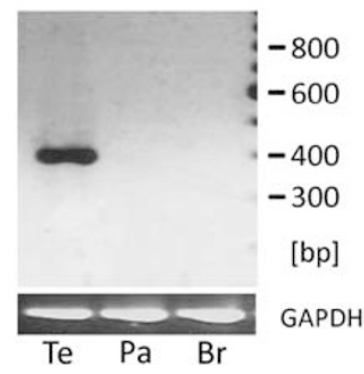

d

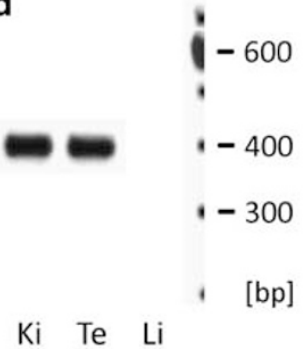

Figure 1 Podocin mRNA expression and sequence analysis reveals the existence of a podocin isoform and proves singular podocin extrarenal expression in human and murine testis. (a) Two podocin isoforms (Podo-1 and Podo-2) could be detected by RT-PCR analysis of kidney fetal (fet) and adult (adu) kidney RNA due to alternative splicing of the podocin pre-mRNA (right). The alternative mRNA form, missing $204 \mathrm{bp}$, was generated by skipping exon 5 of the NPHS2 gene, as could be confirmed by sequencing of the corresponding cDNA. GAPDH RNA was selected as an endogenous RNA control (GAPDH), mRNA of a renal clear cell carcinoma (derived from tubular cells) was used as a negative control (-). Lane M, 100 bp DNA ladder. (b) Constant expression of both isoforms; podocin isoform 1 (Podo-1) and podocin isoform 2 (Podo-2) in three different healthy kidneys (1, 2 and 3) demonstrates that isoform 2 is constitutively expressed and is not an allelic variant of the NPHS2 gene. (c) RT-PCR analysis of podocin expression in human testis $(\mathrm{Te})$, pancreas $(\mathrm{Pa})$ and brain (Br). Podocin isoform 1 was only detected in testis. Isoform 2 is not expressed in any of the tissues. Expression of the housekeeping gene GAPDH was used as an internal control as well as a loading control (GAPDH). (d) Detection of podocin isoform 1 in murine kidney (Ki) and testis (Te) demonstrates that isoform 2 is human specific. Podocin expression in the mouse liver served as a negative control (Li).

was 42.2 and $34.4 \mathrm{kDa}$, respectively. Thus, our immoblot analysis was consistent with theoretical data, demonstrating that both podocin isoforms are not significantly modified (eg by glycosylation).

\section{Immunohistochemical Detection Indicates Sertoli Cells to Be the Origin of Testicular Podocin}

The results of the PCR and the immunoblot assays raised the question of the cellular origin of testicular podocin. Therefore, we performed immunohistochemical staining for podocin in testis, Sertoli cell tumor and kidney (as positive control). Staining of healthy adult testis showed an expression of podocin in the seminiferous tubules (Figure 4a and b). The staining pattern suggested that the protein was expressed in the Sertoli cells rather than 
a

N-MERRARSSSRESRGRGGRTPHKENKRAKAERSGGGRGRQEAGPEPSGSGR 50

AGT PGEPRAPAATVVDVDEVRGSGEEGTEVVALLESERPEEGTKSSGLGA 100

CEWLLVLISLLEI IMTFPFS IWECV VVQEYERVI I FRLGHLLPGRAKGP 150

\begin{tabular}{|l} 
GLFFFLPCLDTYHKVDLRLQTLEIPFHEIVTKDMEIMEIDAICYYRMENA \\
200
\end{tabular}

SLLLSSLAHVSKAVQELVQTTMKRLLAHRSLTEILLERKSIAQDAKVALD 250

SVTCIWGIKVERIEIKDVRLPAGLQHSLAVEAEAQRQAKVRMIAAEAEKA 300

ASESLRMAAEILSGT PAAVQLRYLHTLQSLSTEKPSTVVLPLPFDLLNCL 350

SSPSNRTQGSLPFPSPSKPVEPLNPKKKDSPML-C

383

transmembrane

domain (TM)
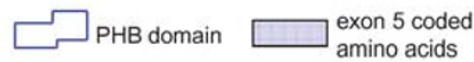

b

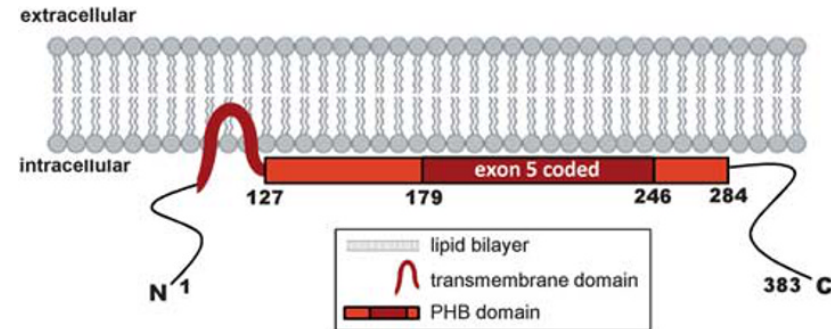

Figure 2 Sequence and membranous localization of podocin. (a) Skipping of the exon 5 (framed in blue) of the NPHS2 gene leads to the translation of a polypeptide lacking the amino acids 179-246 of the isoform 1 (highlighted in blue). Thus, in this isoform 2, the central part of the prohibitin homology domain is missing. Underlined in red is the transmembrane domain (TM). (b) An illustration of the membranous localization of podocin. For the membranous cholesterol binding of podocin, the prohibitin homology domain (amino acids 127-246) (indicated by the arrow) is essential. The loss of the exon 5-coded central core of this domain leads to the inability to bind cholesterol of the inner membrane.

in germ cells. To confirm these results, a Sertoli cell tumor biopsy was stained for podocin showing a homogeneous distribution of the membranous staining pattern along the tumor cells (Figure 4c). Within the seminiferous tubules, the transcription factor WT-1 is exclusively expressed in the nuclei of Sertoli cells and serves as an indicator of testicular Sertoli cell localization (Figure 4b). In consecutive sections of one seminiferous tubule, the WT-1 could be detected within the nuclei of the Sertoli cells and in the same specimen the highly membraneous expression of podocin within the cytoplasm extensions of these cells (Figure 4a and b).

\section{Confocal Laser Scanning Microscopy Demonstrates that Podocin Is Co-localized with F-Actin in the Seminiferous Tubules}

Using confocal laser scanning microscopy, f-actin was stained in the testis, followed by antibody staining of podocin (Figure $5 \mathrm{a}$ and $\mathrm{b}$ ). In the overlay of both stains, confocal laser scanning microscopy was able to detect the co-localization of podocin and f-actin in the human seminiferous tubules (Figure 5d). The overlay of the f-actin, podocin and the nuclear staining showed podocin to be located

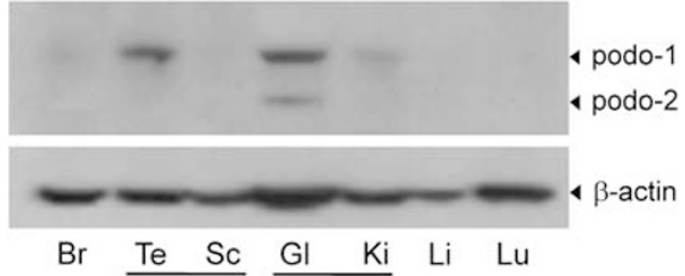

Figure 3 Immunoblot analysis with the $\alpha$-podocin anti-serum p35. Podocin isoform 1 (podo-1) is only expressed in the renal glomeruli (Gl) and in testis (Te), whereas the (shorter) isoform 2 (podo-2) is exclusively expressed in glomeruli. Further organs (brain (Br), liver (Li), lung (Lu), spermatic cords (Sc)) show no expression of either podocin isoform. $\beta$-actin was used as a positive control. Ki, whole kidney.

in the lateral cell membrane of Sertoli cells. Kidney slides were used as a positive control. Staining of the kidney showed specific detection of podocin in the glomeruli (Figure $5 \mathrm{f}$ and $\mathrm{g}$ ) as well as f-actin (Figure 5e).

\section{Real-time Quantification of Podocin and WT-1 mRNA Levels in the Testes of Infertile Men with Sertoli Cell-Only Syndrome}

Pure Sertoli cell-only syndrome is a disorder, characterized by azoospermia, in which the seminiferous tubules contain only Sertoli cells. Thus, we expected an enrichment of podocin mRNA in the testicles of the affected men. To prove this assumption, we used real-time PCR to quantify podocin and WT-1 mRNA expression in normal testes $(n=4)$ and testes of Sertoli cell-only syndrome patients with defective spermatogenesis $(n=5)$. While podocin expression was detected in all normal testes, it was completely absent in all testes of Sertoli cell-only syndrome patients (Figure 6a and c). These data show that podocin is expressed in Sertoli cells of healthy men, but is completely and significantly $(P=0.017)$ absent in the Sertoli cells of men with pure Sertoli cell-only syndrome. In contrast to the podocin expression, the expression of the transcription factor WT-1 is not significantly affected by the disorder. A slight, but not significant, increase in WT-1 transcript levels could be detected in the testes of Sertoli cell-only syndrome patients (Figure $6 b)$. In all RNA samples from patients and healthy men, a positive signal for the $\beta$-2 microglobulin transcript was detected, thus validating the integrity of the RNA preparations.

\section{Discussion}

In this study, we can for the first time, clearly demonstrate an extrarenal expression of podocin and reveal the definite existence of a kidney-specific podocin isoform missing the in-frame exon 5 (now called isoform 2). Although an isoform has been 

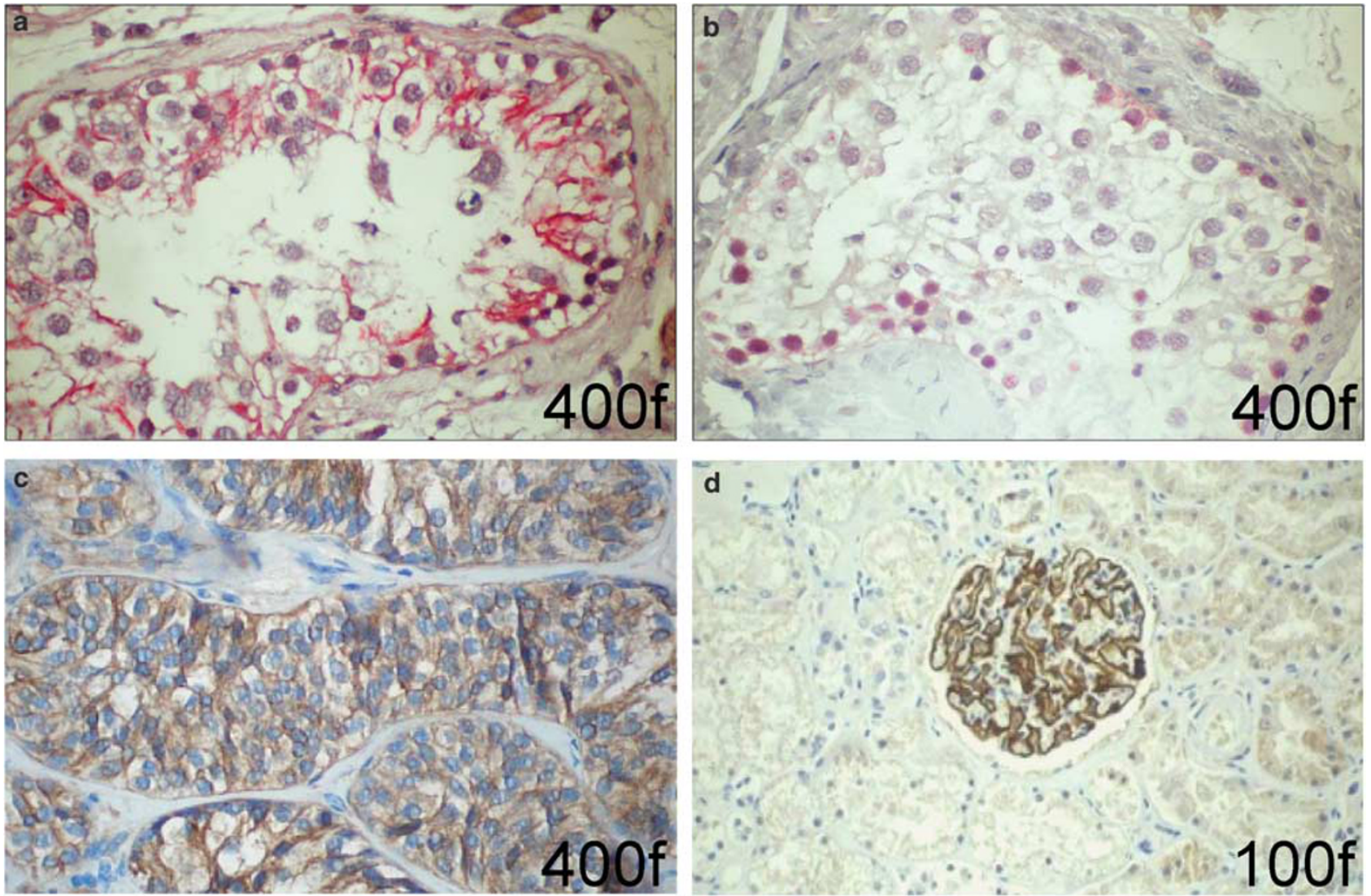

Figure 4 Immunohistochemical detection suggests Sertoli cells to be the origin of testicular podocin. (a, b) Detection of WT-1 in nuclei of Sertoli cells (b) and podocin (a) within the membranes of sertoli cell extensions within a seminiferous tubule (APAAP, $\times 400)$. (c) The same primary antibody, but a biotinylated goat anti-rabbit IgG was used to detect podocin in a Sertoli cell tumor. (d) Podocin expression in renal glomeruli served as a positive control. Original magnification is shown in the lower right-hand corner of each image.

discussed, ${ }^{13}$ until now no direct evidence has been provided.

The skipping of exon 5 leads to a deletion of 68 amino acids, forming the central part of the so-called prohibitin homology domain, which is present in $>340$ different animal proteins. These polypeptides are membrane associated and are capable of regulating homeostasis, mechanosensation and cell signaling. ${ }^{20-22}$ Huber et $a l^{23,24}$ showed that the $\mathrm{PBH}$ domain of podocin is required for membranous cholesterol binding, homo-oligomerization as well as the formation of high-molecular-weight complexes by these homophilic interactions. This finding is consistent with the report that several animal prohibitin homology domain proteins are found in cholesterol-rich membrane fractions derived from the plasma membrane. ${ }^{25}$

Besides its function as a structural protein of the renal filtration unit, podocin is part of a multiprotein complex, consisting of the transmembrane proteins nephrin, neph1, neph2, CD2-associated protein and transient receptor potential channel-6. ${ }^{20}$ This complex anchors the slit diaphragm to the actin cytoskeleton and establishes its location in the lateral plasma membrane of the podocytic foot processes. ${ }^{13}$
In podocytes, nephrin acts as a signal transducer through the phosphorylation of AP- $1{ }^{23}$ Interestingly, AP-1 activation by nephrin is augmented 40 -fold by podocin ${ }^{26}$ and abrogated by a deletion of the prohibitin homology domain of podocin. ${ }^{24}$ Another regulatory target of podocin is the transient receptor potential channel-6, which is co-localized with podocin in podocytes. ${ }^{27}$ In order to regulate this protein, podocin requires the cholesterol binding properties of the prohibitin homology domain. ${ }^{23}$ Isoform 2, lacking this domain, implies a completely different cell and signaling function as the welldescribed podocin. Nonetheless, we are able to prove that both podocin isoforms are simultaneously expressed in healthy human podocytes. At this point, the function and the cellular localization of podocin isoform 2 remain unknown. As the transmembrane domain and the two palmitoylation sites of podocin ${ }^{23}$ are not affected by the deletion of the exon 5-coded amino acids, isoform 2 might be floating within the membrane with the ability to bind other podocin molecules but not nephrin. Of interest are further studies looking into the expression pattern of podocin isoform 2 in patients with nephrotic syndrome.

Besides identifying a novel podocin isoform, we are able to provide evidence of extrarenal podocin 

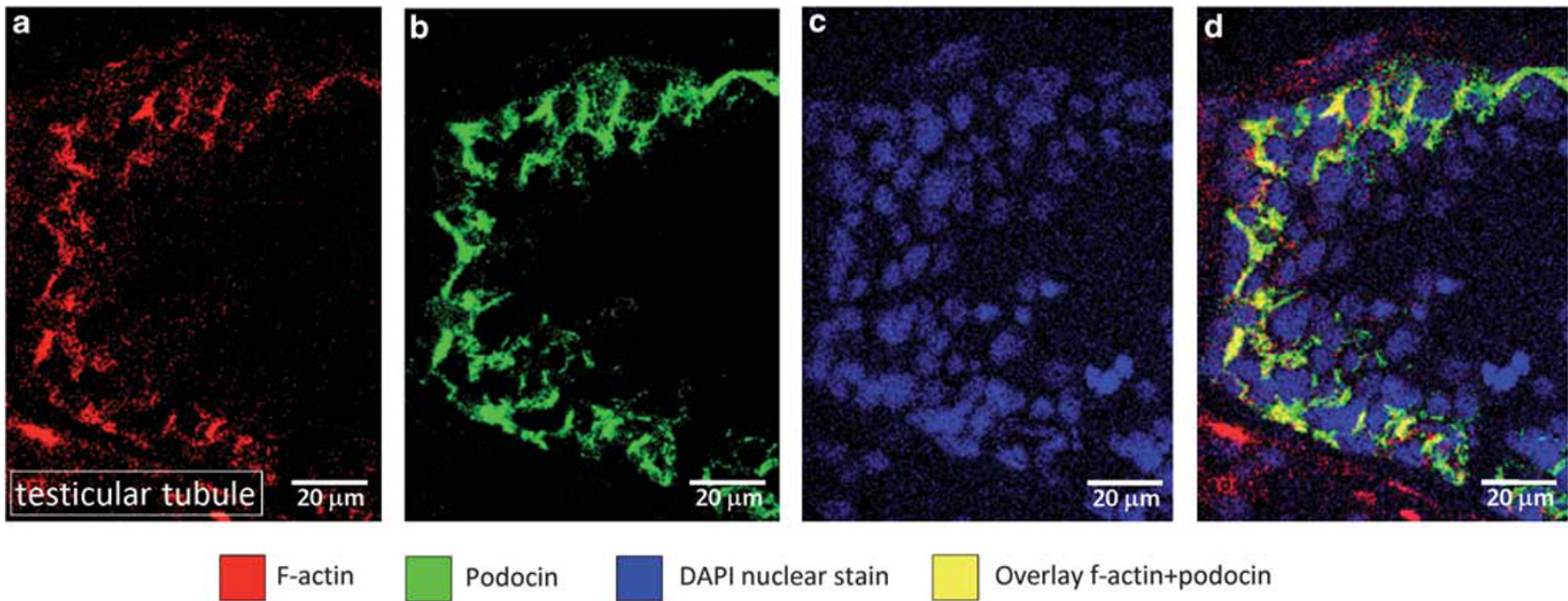

Podocin

DAPI nuclear stain

Overlay f-actin+podocin
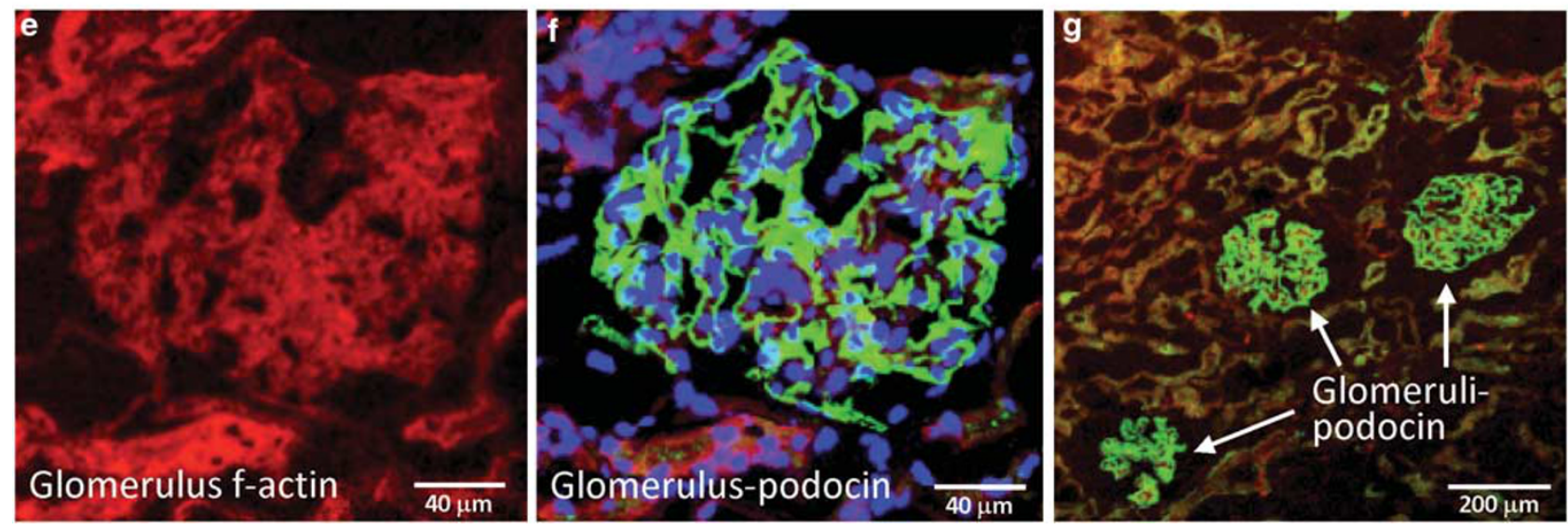

Figure 5 Confocal laser scanning microscopy demonstrates that podocin is co-localized with f-actin in the seminiferous tubules. Podocin (b, green) shows the same expression pattern as f-actin (a, red). Nuclei were counterstained with DAPI (c, blue). The overlay of all these stainings (d) shows that podocin and actin are co-localized (yellow). F-actin (e, red) and podocin (f, g, green) detection in the glomeruli of the kidney were used as positive controls showing the specificity of the antibodies used.

expression. Immunohistochemical staining of healthy human testis as well as biopsies from Sertoli cell tumors revealed a membranous expression of podocin in the seminiferous tubules (Figures 4 and 5), indicating that the Sertoli cells might be the relevant podocin expressing cells in the testis. RT-PCR and immunoblotting data substantiated the immunohistochemical findings. Analogous to the kidney, ${ }^{28}$ testicular podocin is associated with the cytoskeleton and co-localizes with f-actin. Thus, we hypothesize that the podocin expressed in the Sertoli cells might either have a role in the blood/ testis barrier or might also be an integral part of the Sertoli cell crypts, which surround the germ cells. Our data amends the finding that densin, another structural protein of the slit diaphragm, was proven to be expressed in human and murine testis. ${ }^{12}$ Nephrin was shown to be expressed in murine testis, indicating an podocin/nephrin connection in analogy to the kidney. ${ }^{11,12}$ In a study with an inducible nephrin transgene mouse model, male mice showed an absence of the lumen of the seminiferous tubules and a lack of mature spermatids, leading to infertility. ${ }^{29}$

But to this date, extrarenal nephrin expression in human tissue could not be confirmed. ${ }^{30}$ Huber et al ${ }^{23}$ suggested that podocin in podocytes may be part of a mechanosensitive protein complex, as shown in the podocin homolog protein mec-2 of the nematode Caenorhabditis elegans. ${ }^{31}$ In analogy, testicular podocin might be a component of a protein complex, which is involved in the migration of germ cells from the basal to the adluminal compartment of seminiferous tubules. The co-localization of podocin to f-actin indicates podocin's association with the blood/testis barrier. The blood/testis barrier is constituted by the co-existence of tight junctions, ectoplasmic specialization (a testis-specific atypical adherens junction type), desmosome-like junctions and gap junctions. ${ }^{32}$ These junctions function collectively in the maintenance of the barrier integrity present between adjacent Sertoli cells. ${ }^{32,33}$ Besides conferring Sertoli cell polarity and functioning as a barrier by restricting paracellular 
a
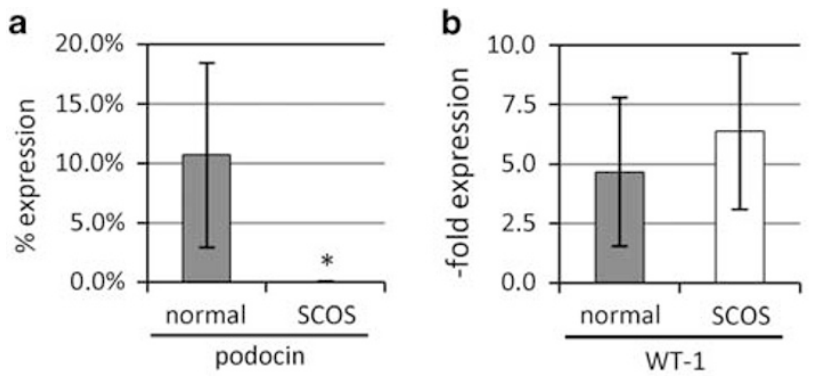

c

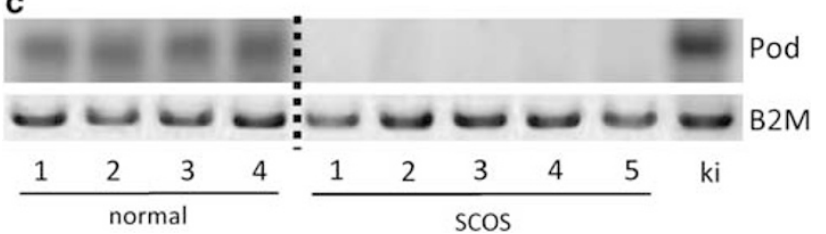

Figure 6 Quantification of podocin mRNA levels in the testes of infertile Sertoli cell-only syndrome patients shows no podocin expression. (a) Quantitative real-time PCR shows a complete down-regulation of podocin in testes of Sertoli cell-only syndrome patients $(n=5)$, (b) whereas the WT-1 expression is slightly, but not significantly, enhanced in the testes of these patients. These data demonstrate that the lack of podocin is not simply a consequence of a WT-1 reduction. mRNA expression was normalized to the housekeeping gene $\beta 2$-microglobulin. (c) Conventional RT-PCR of podocin in normal testes and testes of five Sertoli cell-only syndrome patients, confirming the results shown in (a). Podocin expression in the kidney (ki) was used as a positive control. Data represent means \pm s.e.m. ${ }^{*} P=0.017$; by Mann-Whitney's $U$-test for unpaired data.

transport of, for example, water, ions and electrolytes into the apical compartment, the blood/testis barrier is a highly dynamic structure. During spermatogenesis, spermatocytes must transverse the blood/testis barrier to the adluminal compartment. ${ }^{34}$ At the same time, the blood/testis barrier has to ensure its integrity in order to avoid the production of anti-sperm antibodies. The dynamics of the blood/testis barrier are made possible by a multi-protein complexes at the tight junctions (eg occludin-zonula occludens 1 ) and at the basal ectoplasmic specialization (eg N-cadherin- $\beta$ catenin) associate structurally via an interaction between zonula occludens 1 and $\beta$-catenin. ${ }^{35,36}$ Where podocin is located in the complex structure of the blood/testis barrier remains to be elucidated.

In order to verify the expression of podocin in Sertoli cells, we examined biopsies of patients with pure Sertoli cell-only syndrome, a disorder characterized by the congenital absence of germ cells in the seminiferous tubules. To our surprise, podocin expression could not be detected in testes of Sertoli cell-only syndrome patients. One of the mechanisms leading to azoospermia in Sertoli cell-only syndrome is seen in the disruption of intercellular communication, for example by the loss of the gap junction protein connexin $43 .^{37}$ The pathophysiology of Sertoli cell-only syndrome remains in question. However, patients with Sertoli cell-only syndrome do not develop a renal pathology and the loss of renal podocin expression has not been documented.

It is evident that adequate Sertoli cell function is required for the appropriate differentiation of germ cells. In the vast majority of cases, the specific role played by each protein in connection with germ cell development remains unknown. ${ }^{38}$

Urinary organs and the genital system both originate from the intermediary mesoderm. In the kidney, the Wilms' tumor gene (WT1) is constitutively expressed by the glomerular podocytes. Thus, is used as a specific marker for these cells. ${ }^{1,2}$ As in the kidney, testicular Sertoli cells also continuously express WT- $1 .^{39}$ The two podocyte-specific genes coding for nephrin (NPHS1) and podocalyxin (PODXL) are dramatically down-regulated in mouse models with decreased levels of WT-1, suggesting that these two genes act downstream of WT- $1 .^{40} \mathrm{We}$ were not able to establish a connection between WT-1 expression and the absence of podocin in patients with pure Sertoli cell-only syndrome.

The present results provide direct evidence of a kidney-specific isoform of podocin lacking the prohibitin homology domain, which is co-expressed with isoform 1 in glomerular podocytes. Therefore, our research data introduce a new candidate for the complex structure of the glomerular slit diaphragm, which is likely to have different features than the well-documented isoform 1 . In addition, we reveal the extrarenal expression of podocin in human testis. This finding sheds a new light on the structure of the seminiferous tubules and podocin's potential role in the physiology of the blood/testis barrier. Whether the absence of podocin is of pathophysiological relevance in pure Sertoli cellonly syndrome should to be evaluated in further studies.

\section{Acknowledgements}

We thank Dr Danielle Schild for her expert advice as well as Dr Tom Florian Fuller for critical reading of the manuscript. We also thank Dr Hans Krause for the isolation of total RNA, Professor Corinne Antignac (Université René Descartes, Paris) for generously providing the podocin antiserum p35 and Dr Clemens Cohen for providing glomerular human tissue. We are grateful to Antonio Marin and Magnus Wang for their advice and the prediction of (alternative) splice sites. This work was supported by a center of excellence (SFB548, 'Analysis and modulation of allergies and autoimmune diseases') of the Deutsche Forschungsgemeinschaft (DFG).

\section{Disclosure/conflict of interest}

The authors declare no conflict of interest. 


\section{References}

1 Mundel P, Reiser J, Kriz W. Induction of differentiation in cultured rat and human podocytes. J Am Soc Nephrol 1997;8:697-705.

2 Mundlos S, Pelletier J, Darveau A, et al. Nuclear localization of the protein encoded by the Wilms' tumor gene WT1 in embryonic and adult tissues. Development 1993;119:1329-1341.

3 Morrison AA, Viney RL, Saleem MA, et al. New insights into the function of the Wilms tumor suppressor gene WT1 in podocytes. Am J Physiol Renal Physiol 2008;295:F12-F17.

4 Pavenstadt H, Kriz W, Kretzler M. Cell biology of the glomerular podocyte. Physiol Rev 2003;83:253-307.

5 Kestila M, Lenkkeri U, Mannikko M, et al. Positionally cloned gene for a novel glomerular protein-nephrinis mutated in congenital nephrotic syndrome. Mol Cell 1998;1:575-582.

6 Schwarz K, Simons M, Reiser J, et al. Podocin, a raftassociated component of the glomerular slit diaphragm, interacts with CD2AP and nephrin. J Clin Invest 2001;108:1621-1629.

7 Simons K, Toomre D. Lipid rafts and signal transduction. Nat Rev Mol Cell Biol 2000;1:31-39.

8 Boute N, Gribouval O, Roselli S, et al. NPHS2, encoding the glomerular protein podocin, is mutated in autosomal recessive steroid-resistant nephrotic syndrome. Nat Genet 2000;24:349-354.

9 Niaudet P. Podocin and nephrotic syndrome: implications for the clinician. J Am Soc Nephrol 2004;15: 832-834.

10 Smithies O. Why the kidney glomerulus does not clog: a gel permeation/diffusion hypothesis of renal function. Proc Natl Acad Sci USA 2003;100: 4108-4113.

11 Liu L, Aya K, Tanaka H, et al. Nephrin is an important component of the barrier system in the testis. Acta Med Okayama 2001;55:161-165.

12 Lassila M, Juhila J, Heikkila E, et al. Densin is a novel cell membrane protein of Sertoli cells in the testis. Mol Reprod Dev 2007;74:641-645.

13 Horinouchi I, Nakazato $\mathrm{H}$, Kawano $\mathrm{T}$, et al. In situ evaluation of podocin in normal and glomerular diseases. Kidney Int 2003;64:2092-2099.

14 Brunak S, Engelbrecht J, Knudsen S. Prediction of human mRNA donor and acceptor sites from the DNA sequence. J Mol Biol 1991;220:49-65.

15 Wang M, Marin A. Characterization and prediction of alternative splice sites. Gene 2006;366:219-227.

16 Roselli S, Gribouval O, Boute N, et al. Podocin localizes in the kidney to the slit diaphragm area. Am J Pathol 2002;160:131-139.

17 Thomaidis T, Relle M, Golbas M, et al. Downregulation of alpha-galactosidase A upregulates CD77: functional impact for Fabry nephropathy. Kidney Int 2009;75: 399-407.

18 Relle M, Mayet WJ, Strand D, et al. Proteinase 3/ myeloblastin as a growth factor in human kidney cells. J Nephrol 2003;16:831-840.

19 Jackson IJ. A reappraisal of non-consensus mRNA splice sites. Nucleic Acids Res 1991;19:3795-3798.

20 Benzing T. Signaling at the slit diaphragm. J Am Soc Nephrol 2004;15:1382-1391.

21 Stewart GW. Hemolytic disease due to membrane ion channel disorders. Curr Opin Hematol 2004;11: 244-250.
22 Ernstrom GG, Chalfie M. Genetics of sensory mechanotransduction. Annu Rev Genet 2002;36: 411-453.

23 Huber TB, Schermer B, Muller RU, et al. Podocin and MEC-2 bind cholesterol to regulate the activity of associated ion channels. Proc Natl Acad Sci USA 2006;103:17079-17086.

24 Huber TB, Simons M, Hartleben B, et al. Molecular basis of the functional podocin-nephrin complex: mutations in the NPHS2 gene disrupt nephrin targeting to lipid raft microdomains. Hum Mol Genet 2003; 12:3397-3405.

25 Morrow IC, Parton RG. Flotillins and the PHB domain protein family: rafts, worms and anaesthetics. Traffic 2005;6:725-740.

26 Huber TB, Kottgen M, Schilling B, et al. Interaction with podocin facilitates nephrin signaling. J Biol Chem 2001;276:41543-41546.

27 Reiser J, Polu KR, Moller CC, et al. TRPC6 is a glomerular slit diaphragm-associated channel required for normal renal function. Nat Genet 2005;37: 739-744.

28 Saleem MA, Ni L, Witherden I, et al. Co-localization of nephrin, podocin, and the actin cytoskeleton: evidence for a role in podocyte foot process formation. Am J Pathol 2002;161:1459-1466.

29 Juhila J, Lassila M, Roozendaal R, et al. Inducible nephrin transgene expression in podocytes rescues nephrin-deficient mice from perinatal death. Am J Pathol 2010;176:51-63.

30 Kuusniemi AM, Kestila M, Patrakka J, et al. Tissue expression of nephrin in human and pig. Pediatr Res 2004;55:774-781.

31 Huang M, Gu G, Ferguson EL, et al. A stomatin-like protein necessary for mechanosensation in C. elegans. Nature 1995;378:292-295.

32 Yan HH, Mruk DD, Lee WM, et al. Ectoplasmic specialization: a friend or a foe of spermatogenesis? Bioessays 2007;29:36-48.

33 Lee NP, Cheng CY. Ectoplasmic specialization, a testis-specific cell-cell actin-based adherens junction type: is this a potential target for male contraceptive development? Hum Reprod Update 2004;10: 349-369.

34 Russell L. Movement of spermatocytes from the basal to the adluminal compartment of the rat testis. Am J Anat 1977;148:313-328.

35 Yan HH, Cheng CY. Blood-testis barrier dynamics are regulated by an engagement/disengagement mechanism between tight and adherens junctions via peripheral adaptors. Proc Natl Acad Sci USA 2005;102: 11722-11727.

36 Siu MK, Cheng CY. Extracellular matrix: recent advances on its role in junction dynamics in the seminiferous epithelium during spermatogenesis. Biol Reprod 2004;71:375-391.

37 Defamie N, Berthaut I, Mograbi B, et al. Impaired gap junction connexin43 in Sertoli cells of patients with secretory azoospermia: a marker of undifferentiated Sertoli cells. Lab Invest 2003;83:449-456.

38 Petersen C, Soder O. The sertoli cell-a hormonal target and 'super' nurse for germ cells that determines testicular size. Horm Res 2006;66:153-161.

39 Sharpe RM, McKinnell C, Kivlin C, et al. Proliferation and functional maturation of Sertoli cells, and their relevance to disorders of testis function in adulthood. Reproduction 2003;125:769-784. 
40 Guo JK, Menke AL, Gubler MC, et al. WT1 is a key regulator of podocyte function: reduced expression levels cause crescentic glomerulonephritis and mesangial sclerosis. Hum Mol Genet 2002;11: 651-659.
This work is licensed under the Creative

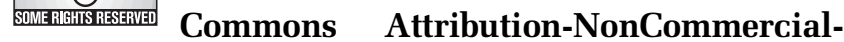
No Derivative Works 3.0 Unported License. To view a copy of this license, visit http://creativecommons. org/licenses/by-nc-nd/3.0/

Supplementary Information accompanies the paper on Modern Pathology website (http://www.nature.com/ modpathol) 